\title{
GRADIENT ESTIMATES AND DOMAIN IDENTIFICATION FOR ANALYTIC ORNSTEIN-UHLENBECK OPERATORS
}

\author{
JAN MAAS AND JAN VAN NEERVEN
}

Dedicated to Herbert Amann on the occasion of his 70th birthday

\begin{abstract}
Let $P$ be the Ornstein-Uhlenbeck semigroup associated with the stochastic Cauchy problem

$$
d U(t)=A U(t) d t+d W_{H}(t)
$$

where $A$ is the generator of a $C_{0}$-semigroup $S$ on a Banach space $E, H$ is a Hilbert subspace of $E$, and $W_{H}$ is an $H$-cylindrical Brownian motion. Assuming that $S$ restricts to a $C_{0}$-semigroup on $H$, we obtain $L^{p}$-bounds for $D_{H} P(t)$. We show that if $P$ is analytic, then the invariance assumption is fulfilled. As an application we determine the $L^{p}$-domain of the generator of $P$ explicitly in the case where $S$ restricts to a $C_{0}$-semigroup on $H$ which is similar to an analytic contraction semigroup. The results are applied to the $1 \mathrm{D}$ stochastic heat equation driven by additive space-time white noise.
\end{abstract}

\section{INTRODUCTION}

Consider the stochastic Cauchy problem

$$
\begin{aligned}
d U(t) & =A U(t) d t+d W_{H}(t), \quad t \geqslant 0, \\
U(0) & =x .
\end{aligned}
$$

Here $A$ generates a $C_{0}$-semigroup $S=(S(t))_{t \geqslant 0}$ on a real Banach space $E, H$ is a real Hilbert subspace continuously embedded in $E, W_{H}$ is an $H$-cylindrical Brownian motion on a probability space $(\Omega, \mathscr{F} P)$, and $x \in E$. A weak solution is a measurable adapted $E$-valued process $U^{x}=\left(U^{x}(t)\right)_{t \geqslant 0}$ such that $t \mapsto U^{x}(t)$ is integrable almost surely and for all $t \geqslant 0$ and $x^{*} \in \mathrm{D}\left(A^{*}\right)$ one has

$$
\left\langle U^{x}(t), x^{*}\right\rangle=\left\langle x, x^{*}\right\rangle+\int_{0}^{t}\left\langle U^{x}(s), A^{*} x^{*}\right\rangle d s+W_{H}(t) i^{*} x^{*} \text { almost surely. }
$$

Here $i: H \hookrightarrow E$ is the inclusion mapping. A necessary and sufficient condition for the existence of a weak solution is that the operators $I_{t}: L^{2}(0, t ; H) \rightarrow E$,

$$
I_{t} g:=\int_{0}^{t} S(s) i g(s) d s
$$

1991 Mathematics Subject Classification. 47D07 (35R15, 42B25, 60H15).

Key words and phrases. Ornstein-Uhlenbeck operators, gradient estimates, domain identification.

The authors are supported by VIDI subsidy 639.032.201 (JM) and VICI subsidy 639.033.604 $(\mathrm{JvN})$ of the Netherlands Organisation for Scientific Research (NWO). 
are $\gamma$-radonifying for all $t \geqslant 0$. If this is the case, then $s \mapsto S(t-s) i$ is stochastically integrable on $(0, t)$ with respect to $W_{H}$ and the process $U^{x}$ is given by

$$
U^{x}(t)=S(t) x+\int_{0}^{t} S(t-s) i d W_{H}(s), \quad t \geqslant 0 .
$$

For more information and an explanation of the terminology we refer to [30].

Assuming the existence of the solution $U^{x}$, on the Banach space $C_{\mathrm{b}}(E)$ of all bounded continuous functions $f: E \rightarrow \mathbb{R}$ one defines the Ornstein-Uhlenbeck semigroup $P=(P(t))_{t \geqslant 0}$ by

$$
P(t) f(x):=\mathbb{E} f\left(U^{x}(t)\right), \quad t \geqslant 0, x \in E .
$$

The operators $P(t)$ are linear contractions on $C_{\mathrm{b}}(E)$ and satisfy $P(0)=I$ and $P(s) P(t)=P(s+t)$ for all $s, t \geqslant 0$. For all $f \in C_{\mathrm{b}}(E)$ the mapping $(t, x) \mapsto$ $P(t) f(x)$ is continuous, uniformly on compact subsets of $[0, \infty) \times E$.

If the operator $I_{\infty}: L^{2}(0, \infty ; H) \rightarrow E$ defined by

$$
I_{\infty} g:=\int_{0}^{\infty} S(t) i g(t) d t
$$

is $\gamma$-radonifying, then the problem (SCP admits a unique invariant measure $\mu_{\infty}$. This measure is a centred Gaussian Radon measure on $E$, and its covariance operator equals $I_{\infty} I_{\infty}^{*}$. Throughout this paper we shall assume that this measure exists; if (SCP) has a solution, then this assumption is for instance fulfilled if $S$ is uniformly exponentially stable. The reproducing kernel Hilbert space associated with $\mu_{\infty}$ is denoted by $H_{\infty}$. The inclusion mapping $H_{\infty} \hookrightarrow E$ is denoted by $i_{\infty}$. Recall that $Q_{\infty}:=i_{\infty} i_{\infty}^{*}=I_{\infty} I_{\infty}^{*}$. Is is well-known that $S$ restricts to a $C_{0}$-contraction semigroup on $H_{\infty}$ [5] (the proof for Hilbert spaces $E$ extends without change to Banach spaces $E$ ), which we shall denote by $S_{\infty}$.

By a standard application of Jensen's inequality, the semigroup $P$ has a unique extension to a $C_{0}$-contraction semigroup to the spaces $L^{p}(E, \mu 1), 1 \leqslant p<\infty$. By slight abuse of notation we shall denote this semigroup by $P$ again. Its infinitesimal generator will be denoted by $L$. In order to give an explicit expression for $L$ it is useful to introduce, for integers $k, l \geqslant 0$, the space $\mathscr{F} C_{\mathrm{b}}^{k, l}(E)$ consisting of all functions $f \in C_{\mathrm{b}}(E)$ of the form

$$
f(x)=\varphi\left(\left\langle x, x_{1}^{*}\right\rangle, \ldots,\left\langle x, x_{N}^{*}\right\rangle\right)
$$

with $f \in C_{\mathrm{b}}^{k}\left(\mathbb{R}^{N}\right)$ and $x_{1}^{*}, \ldots, x_{N}^{*} \in \mathrm{D}\left(A^{* l}\right)$. With this notation one has that $\mathscr{F} C_{\mathrm{b}}^{2,1}(E)$ is a core for $L$, and on this core one has

$$
L f(x)=\frac{1}{2} \operatorname{tr} D_{H}^{2} f(x)+\left\langle x, A^{*} D f(x)\right\rangle .
$$

Here,

$$
\begin{aligned}
D_{H} f(x) & =\sum_{n=1}^{N} \frac{\partial \varphi}{\partial x_{n}}\left(\left\langle x, x_{1}^{*}\right\rangle, \ldots,\left\langle x, x_{N}^{*}\right\rangle\right) \otimes i^{*} x_{n}^{*}, \\
D f(x) & =\sum_{n=1}^{N} \frac{\partial \varphi}{\partial x_{n}}\left(\left\langle x, x_{1}^{*}\right\rangle, \ldots,\left\langle x, x_{N}^{*}\right\rangle\right) \otimes x_{n}^{*},
\end{aligned}
$$

denote the Fréchet derivatives into the directions of $H$ and $E$, respectively. 


\section{Gradient estimates: the $H$-inVARiant CASE}

Our first result gives a pointwise gradient bound for $P$ under the assumption that $S$ restricts to a $C_{0}$-semigroup on $H$ which will be denoted by $S_{H}$. As has been shown in [17, Corollary 5.6], under this assumption the operator $D_{H}$ is closable as a densely defined operator from $L^{p}(E, \mu 1)$ to $L^{p}(E, \mu ; H)$ for all $1 \leqslant p<\infty$. The domain of its closure is denoted by $\mathrm{D}_{p}\left(D_{H}\right)$.

Proposition 2.1 (Pointwise gradient bounds). If $S$ restricts to a $C_{0}$-semigroup on $H$, then for all $1<p<\infty$ there exists a constant $C \geqslant 0$ such that for all $t>0$ and $f \in \mathscr{F} C_{\mathrm{b}}^{1,0}(E)$ we have

$$
\sqrt{t}\left|D_{H} P(t) f(x)\right| \leqslant C \kappa(t)\left(P(t)|f|^{p}(x)\right)^{1 / p},
$$

where $\kappa(t):=\sup _{s \in[0, t]}\left\|S_{H}(s)\right\|_{E(H)}$.

Proof. The proof follows the lines of [25, Theorem 8.10] and is inspired by the proof of [10, Theorem 6.2.2], where the null controllable case was considered.

The distribution $\mu_{t}$ of the random variable $U^{0}(t)$ is a centred Gaussian Radon measure on $E$. Let $H_{t}$ denote its RKHS and let $i_{t}: H_{t} \hookrightarrow E$ be the inclusion mapping. As is well known and easy to prove, cf. [9, Appendix B] one has

$$
H_{t}=\left\{\int_{0}^{t} S(t-s) i g(s) d s: g \in L^{2}(0, t ; H)\right\}
$$

with

$$
\|h\|_{H_{t}}=\inf \left\{\|g\|_{L^{2}(0, t ; H)}: h=\int_{0}^{t} S(t-s) i g(s) d s\right\} .
$$

The mapping

$$
\phi^{\mu_{t}}: i_{t}^{*} x^{*} \mapsto\left\langle\cdot, x^{*}\right\rangle, \quad x^{*} \in E^{*},
$$

defines an isometry from $H_{t}$ onto a closed subspace of $L^{2}\left(E, \mu_{t}\right)$. For $h \in H_{t}$ we shall write $\phi_{h}^{\mu_{t}}(x):=\left(\phi^{\mu_{t}} h\right)(x)$.

Fix $h \in H$. Since $S$ restricts to a $C_{0}$-semigroup $S_{H}$ on $H$ we may consider the function $g \in L^{2}(0, t ; H)$ given by $g(s)=\frac{1}{t} S(s) h$. From the identity $S(t) h=$ $\int_{0}^{t} S(t-s) g(s) d s$ we deduce that $S(t) h \in H_{t}$ and

$$
\|S(t) h\|_{H_{t}}^{2} \leqslant\|g\|_{L^{2}(0, t ; H)}^{2}=\frac{1}{t^{2}} \int_{0}^{t}\|S(s) h\|_{H}^{2} d s \leqslant \frac{1}{t} \kappa(t)^{2}\|h\|_{H}^{2} .
$$

Fix a function $f \in \mathscr{F} C_{\mathrm{b}}^{1,0}(E)$, that is, $f(x)=\varphi\left(\left\langle x, x_{1}^{*}\right\rangle, \ldots,\left\langle x, x_{N}^{*}\right\rangle\right)$ with $\varphi \in$ $C_{\mathrm{b}}^{1}\left(\mathbb{R}^{N}\right)$ and $x_{1}^{*}, \ldots, x_{N}^{*} \in E^{*}$. It is easily checked that for all $t>0$ we have $P(t) f \in$ $\mathscr{F} C_{\mathrm{b}}^{1,0}(E)$; in particular this implies that $P(t) f \in \mathrm{D}_{p}\left(D_{H}\right)$. By the CameronMartin formula [3],

$$
\begin{aligned}
\frac{1}{\varepsilon}(P(t) f(x+\varepsilon h)-P(t) f(x)) & =\frac{1}{\varepsilon} \int_{E}(f(S(t)(x+\varepsilon h)+y)-f(S(t) x+y)) d \mu_{t}(y) \\
& =\int_{E} \frac{1}{\varepsilon}\left(E_{\varepsilon S(t) h}-1\right) f(S(t) x+y) d \mu_{t}(y)
\end{aligned}
$$

where for $h \in H_{t}$ we write

$$
E_{h}(x):=\exp \left(\phi_{h}^{\mu_{t}}(x)-\frac{1}{2}\|h\|_{H_{t}}^{2}\right) .
$$


It is easy to see that for each $h \in H_{t}$ the family $\left(\frac{1}{\varepsilon}\left(E_{\varepsilon h}-1\right)\right)_{0<\varepsilon<1}$ is uniformly bounded in $L^{2}\left(E, \mu_{t}\right)$, and therefore uniformly integrable in $L^{1}\left(E, \mu_{t}\right)$. Passage to the limit $\varepsilon \downarrow 0$ in the previous identity now gives

$$
\left[D_{H} P(t) f(x), h\right]=\int_{E} f(S(t) x+y) \phi_{S(t) h}^{\mu_{t}}(y) d \mu_{t}(y) .
$$

By Hölder's inequality with $\frac{1}{r}+\frac{1}{q}=1$ and the Kahane-Khintchine inequality, which can be applied since $\phi_{S(t) h}^{\mu_{t}}$ is a Gaussian random variable,

$$
\begin{aligned}
\mid\left[D_{H}\right. & P(t) f(x), h] \mid \\
& \leqslant\left(\int_{E}|f(S(t) x+y)|^{r} d \mu_{t}(y)\right)^{\frac{1}{r}}\left(\int_{E}\left|\phi_{S(t) h}^{\mu_{t}}(y)\right|^{q} d \mu_{t}(y)\right)^{\frac{1}{q}} \\
& \leqslant K_{q}\left(\int_{E}|f(S(t) x+y)|^{r} d \mu_{t}(y)\right)^{\frac{1}{r}}\left(\int_{E}\left|\phi_{S(t) h}^{\mu_{t}}(y)\right|^{2} d \mu_{t}(y)\right)^{\frac{1}{2}} \\
& =K_{q}\left(P(t)|f|^{r}(x)\right)^{\frac{1}{r}}\|S(t) h\|_{H_{t}} .
\end{aligned}
$$

Using (2.1) we find that

$$
\left|\sqrt{t}\left[D_{H} P(t) f(x), h\right]\right| \leqslant K_{q} \kappa(t)\left(P(t)|f|^{r}(x)\right)^{\frac{1}{r}}\|h\|_{H},
$$

and by taking the supremum over all $h \in H$ of norm 1 we obtain the desired estimate.

Corollary 2.2. If $S$ restricts to a $C_{0}$-semigroup on $H$, then for all $1<p<\infty$ the operators $D_{H} P(t), t>0$, extend uniquely to bounded operators from $L^{p}(E, \mu 1)$ to $L^{p}(E, \mu 1 ; H)$, and there exists a constant $C \geqslant 0$ such that for any $t>0$,

$$
\sqrt{t}\left\|D_{H} P(t)\right\|_{\mathscr{L}\left(L^{p}(E, \mu 1), L^{p}(E, \mu 1 ; H)\right)} \leqslant C \kappa(t) .
$$

Proof. Integrating the inequality of the proposition and using the fact that $\mu_{\infty}$ is an invariant measure for $P$ we obtain

$$
\begin{aligned}
\left\|\sqrt{t} D_{H} P(t) f\right\|_{L^{p}(E, \mu 1)}^{p} & \leqslant C^{p} \kappa(t)^{p} \int_{E} P(t)|f|^{p}(x) d \mu 1(x) \\
& =C^{p} \kappa(t)^{p} \int_{E}|f|^{p}(x) d \mu 1(x)=C^{p} \kappa(t)^{p}\|f\|_{L^{p}(E, \mu 1)}^{p}
\end{aligned}
$$

\section{Gradient estimates: the Analytic Case}

Analyticity of the semigroup $P$ on $L^{p}\left(E, \mu_{\infty}\right)$ has been investigated by several authors [15, 16, 18, 24]. The following result of [18 is our starting point. Recall that in the definition of an analytic $C_{0}$-contraction semigroup, contractivity is required on an open sector containing the positive real axis.

Proposition 3.1. For any $1<p<\infty$ the following assertions are equivalent:

(1) $P$ is an analytic $C_{0}$-semigroup on $L^{p}\left(E, \mu_{\infty}\right)$;

(2) $P$ is an analytic $C_{0}$-contraction semigroup on $L^{p}\left(E, \mu_{\infty}\right)$;

(3) $S$ restricts to an analytic $C_{0}$-contraction semigroup on $H_{\infty}$;

(4) $Q_{\infty} A^{*}$ acts as a bounded operator in $H$. 
A more precise formulation of (4) is that there should exist a bounded operator $B: H \rightarrow H$ such that $i B i^{*} x^{*}=Q_{\infty} A^{*} x^{*}$ for all $x^{*} \in E^{*}$. The identity $Q_{\infty} A^{*}+$ $A Q_{\infty}=-i i^{*}$ implies that $B+B^{*}=-I$.

In what follows we shall simply say that ' $P$ is analytic' to express that the equivalent conditions of the proposition are satisfied for some (and hence for all) $1<p<\infty$.

The next result has been shown in [24] for $p=2$ and was extended to $1<p<\infty$ in [25].

Proposition 3.2. If $P$ is analytic, then $\mathscr{F} C_{\mathrm{b}}^{2,1}(E)$ is a core for the generator $L$ of $P$ in $L^{p}\left(E, \mu_{\infty}\right)$, and on this core $L$ is given by

$$
L=D_{H}^{*} B D_{H} \text {. }
$$

Our first aim is to show that analyticity of $P$ implies that $H$ is $S$-invariant. For self-adjoint $P$ this was proved in [7, 18.

Theorem 3.3. If $P$ is analytic, then $S$ restricts to a bounded analytic $C_{0}$-semigroup $S_{H}$ on $H$

Proof. Consider the linear mapping

$$
V: i 1^{*} x^{*} \mapsto i^{*} x^{*}, \quad x^{*} \in E^{*} .
$$

It is shown in 17 that $i_{1}^{*} x^{*}=0$ implies $i^{*} x^{*}=0$, so that this mapping is welldefined, and that the closability of $D_{H}$ implies the closability of $V$ as a densely defined operator from $H_{\infty}$ to $H$. With slight abuse of notation we denote its closure by $V$ again and let $\mathrm{D}(V)$ the domain of the closure.

By [1, Proposition 7.1], the operator $-V V^{*} B$ is sectorial of angle $<\frac{\pi}{2}$, and therefore $G:=V V^{*} B$ generates a bounded analytic $C_{0}$-semigroup $(T(t))_{t \geq 0}$ on $H$. To prove the theorem, by uniqueness of analytic continuation and duality it suffices to show that $T(t) \circ i^{*}=i^{*} \circ S^{*}(t)$ for all $t \geqslant 0$.

For all $x^{*} \in \mathrm{D}\left(A^{*}\right)$ we have $B i^{*} x^{*} \in \mathrm{D}\left(V^{*}\right)$ and $V^{*} B i^{*} x^{*}=i_{\infty}^{*} A^{*} x^{*}$. Indeed, for $y^{*} \in E^{*}$ one has

$$
\left[B i^{*} x^{*}, V i_{\infty}^{*} y^{*}\right]=\left\langle i_{\infty}^{*} A^{*} x^{*}, i_{\infty}^{*} y^{*}\right\rangle,
$$

which implies the claim. By applying the operator $V$ to this identity we obtain $i^{*} x^{*} \in \mathrm{D}(G)$ and $G i^{*} x^{*}=i^{*} A^{*} x^{*}$, from which it follows that $T(t) i^{*} x^{*}=i^{*} S^{*}(t) x^{*}$. This proves the theorem, with $S_{H}=T^{*}$.

This result should be compared with [18, Theorem 9.2], where it is shown that if $S$ restricts to an analytic $C_{0}$-semigroup on $H$ which is contractive in some equivalent Hilbert space norm, then $P$ is analytic on $L^{p}(E, \mu 1)$.

Under the assumption that $P$ is analytic on $L^{p}(E, \mu 1)$, the gradient estimates of the previous section can be improved as follows. Recall that a collection of bounded operators $\mathscr{T}$ between Banach spaces $X$ and $Y$ is said to be $R$-bounded if there exists a constant $C$ such that for any finite subset $T_{1}, \ldots, T_{n} \subset \mathscr{T}$ and any $x_{1}, \ldots, x_{n} \in X$ we have

$$
\mathbb{E}\left\|\sum_{j=1}^{n} r_{j} T_{j} x_{j}\right\|^{2} \leqslant C^{2} \mathbb{E}\left\|\sum_{j=1}^{n} r_{j} x_{j}\right\|^{2}
$$

where $\left(r_{j}\right)_{j \geqslant 1}$ is an independent collection of Rademacher random variables. The notion of $R$-boundedness plays an important role in recent advances in the theory of evolution equations (see [12, 21]). 
Theorem 3.4. If $P$ is analytic, then for all $1<p<\infty$ the set

$$
\left\{\sqrt{t} D_{H} P(t): t>0\right\}
$$

is $R$-bounded in $\mathscr{L}\left(L^{p}(E, \mu 1), L^{p}(E, \mu 1 ; H)\right)$ and we have the square function estimate

$$
\left\|\left(\int_{0}^{t}\left\|D_{H} P(t) f\right\|_{H}^{2} d t\right)^{1 / 2}\right\|_{L^{p}(E, \mu 1)} \lesssim\|f\|_{L^{p}(E, \mu 1)}
$$

with implied constant independent of $f \in L^{p}(E, \mu 1)$.

Proof. By Proposition 3.2 and Theorem 3.3 , the theorem is a special case of [25], Theorem 2.2].

The above result plays a crucial role in our recent paper 25] in which $L^{p}$-domain characterisations for the operator $L$ and its square root have been obtained. Before stating the result, let us informally sketch how Theorem 3.4 enters the argument. In order to prove a domain characterisation for the operator $L$, we first aim to obtain two-sided estimates for $\|\sqrt{-L} f\|_{L^{p}\left(E, \mu_{\infty}\right)}$ in terms of suitable Sobolev norms. For this purpose we consider a variant of an operator theoretic framework introduced in [2] in the analysis of the famous Kato square root problem. The idea behind this framework is that the second order operator $L$ can be naturally studied through the first order Hodge-Dirac-type operator

$$
\Pi=\left[\begin{array}{cc}
0 & -D_{H}^{*} B \\
D_{H} & 0
\end{array}\right] \text { on } L^{p}\left(E, \mu_{\infty}\right) \oplus L^{p}\left(E, \mu_{\infty} ; H\right) .
$$

This operator is bisectorial and its square is the sectorial operator given by

$$
-\Pi^{2}=\left[\begin{array}{cc}
D_{V}^{*} B D_{V} & 0 \\
0 & D_{V} D_{V}^{*} B
\end{array}\right]=\left[\begin{array}{cc}
L & 0 \\
0 & \underline{L}
\end{array}\right],
$$

where $\underline{L}:=D_{V} D_{V}^{*} B$. The approach in $[25$ ] consists of proving estimates for $\sqrt{-L} f$ along the lines of the following formal calculation:

$$
\left\|D_{H} f\right\|_{p}=\|\Pi(f, 0)\|_{p} \leqslant\left\|\Pi / \sqrt{\Pi^{2}}\right\|_{p}\left\|\sqrt{\Pi^{2}}(f, 0)\right\|_{p}=\left\|\Pi / \sqrt{\Pi^{2}}\right\|_{p}\|\sqrt{L} f\|_{p} .
$$

Oversimplifying things considerably, the proof consists of turning this calculation into rigourous mathematics. This can be done once we know that the operator $\Pi / \sqrt{\Pi^{2}}$ is bounded. Since the function $z \mapsto z / \sqrt{z^{2}}$ is a bounded analytic function on each bisector around the real axis, it suffices to show that $\Pi$ has a bounded $H^{\infty}$-functional calculus. This in turn will follow if we show that

(1) the resolvent set $\left\{(i t-\Pi)^{-1}\right\}_{t \in \mathbb{R} \backslash\{0\}}$ is $R$-bounded;

(2) the operator $\Pi^{2}$ admits a bounded functional calculus.

To prove (1), we observe that

$$
(I-i t \Pi)^{-1}=\left[\begin{array}{cc}
\left(1-t^{2} L\right)^{-1} & -i t\left(I-t^{2} L\right)^{-1} D_{H}^{*} B \\
i t D_{H}\left(I-t^{2} L\right)^{-1} & \left(I-t^{2} \underline{L}\right)^{-1}
\end{array}\right], \quad t \in \mathbb{R} \backslash\{0\} .
$$

It suffices to prove $R$-boundedness for each of the entries separately. The diagonal entries can be dealt with using abstract results on $R$-boundedness for positive contraction semigroups on $L^{p}$-spaces. The $R$-boundedness for the off-diagonal entries can be derived using Theorem 3.4

To prove (2) we use the fact that the semigroup generated by $\underline{L}$ equals $P \otimes S_{H}^{*}$ on the range of the gradient $D_{H}$. Here $S_{H}$ denotes the restriction of the semigroup 
$S$ to $H$ (see Theorem 3.3). Therefore (2) follows, provided that the negative generator $-A_{H}$ of $S_{H}$ has a bounded $H^{\infty}$-calculus. This reduces the original question about $\sqrt{-L}$ to a question about the operator $A_{H}$, which is defined directly in terms of the data $H$ and $A$ of the problem. The latter question should be thought of as expressing the compatibility of the drift (represented by the operator $A$ ) and the noise (represented by the Hilbert space $H$ ). This compatibility condition is not automatically satisfied. In fact, by a result of Le Merdy [22, $-A_{H}$ admits a bounded $H^{\infty}$-functional calculus on $H$ if and only if $S_{H}$ is an analytic $C_{0^{-}}$-contraction semigroup on $H$ with respect to some equivalent Hilbert space norm. Such needs not always be the case, as is shown by well-known examples [26].

The following result summarises the informal discussion above and provides an additional equivalent condition in terms of the operator $A_{\infty}$. In this result we let $\mathrm{D}_{p}\left(D_{H}^{2}\right)$ denote the second order Sobolev space associated with the operator $D_{H}$.

Theorem 3.5. Let $1<p<\infty$. If $P$ is analytic on $L^{p}(E, \mu 1)$, then the following assertions are equivalent:

(1) $\mathrm{D}_{p}(\sqrt{-L})=\mathrm{D}_{p}\left(D_{H}\right)$ with norm equivalence

$$
\|\sqrt{-L} f\|_{L^{p}(E, \mu 1)} \bar{\sim}\left\|D_{H} f\right\|_{L^{p}(E, \mu 1 ; H)} ;
$$

(2) $\mathrm{D}\left(\sqrt{-A_{\infty}}\right)=\mathrm{D}(V)$ with norm equivalence

$$
\|\sqrt{-A} h\|_{H_{\infty}} \approx\|V h\|_{H}
$$

(3) $-A_{H}$ admits a bounded $H^{\infty}$-functional calculus on $H$.

If these equivalent conditions are satisfied we have

$$
\mathrm{D}_{p}(L)=\mathrm{D}_{p}\left(D_{H}^{2}\right) \cap \mathrm{D}_{p}\left(A_{\infty}^{*} D\right),
$$

where $D$ is the Malliavin derivative in the direction of $H_{\infty}$.

Proof. By Proposition 3.2 and Theorem 3.3 , the theorem is a special case of [25, Theorems 2.1, 2.2] provided we replace $A_{\infty}$ by $A_{\infty}^{*}$ in (2). The equivalence of (2) for $A_{\infty}$ and $A_{\infty}^{*}$, however, is well known (see also [25, Lemma 10.2]).

The problem of identifying the domains of $\sqrt{-L}$ and $L$ has a long and interesting history. We finish this paper by presenting three known special cases of Theorem 3.5. In each case, it is easy to verify that (3) is satisfied.

Example 1. For the classical Ornstein-Uhlenbeck operator, which corresponds to $H=E=\mathbb{R}^{d}$ and $A=-I$, conditions (2) and (3) of Theorem [3.5 are trivially fulfilled and (1) reduces to the classical Meyer inequalities of Malliavin calculus. For a discussion of Meyer's inequalities we refer to the book of Nualart 31.

Example 2. Meyer's inequalities were extended to infinite dimensions by Shigekawa 32, and Chojnowska-Michalik and Goldys [6, 7], who considered the case where $E$ is a Hilbert space and $A_{H}$ is self-adjoint. Both authors deduce the generalised Meyer inequalities from square functions estimates. The identification of $\mathrm{D}_{p}(L)$ in the self-adjoint case is due to Chojnowska-Michalik and Goldys 6, 7, who extended the case $p=2$ obtained earlier by Da Prato $[\underline{8}$.

So far, these examples were concerned with the selfadjoint case. 
Example 3. A non-selfadjoint extension of Meyer's inequalities has been given for the case $E=\mathbb{R}^{d}$ by Metafune, Prüss, Rhandi, and Schnaubelt 27] under the nondegeneracy assumption $H=\mathbb{R}^{d}$. In this situation the semigroup $P$ is analytic on $L^{p}\left(\mu_{\infty}\right)$ [15, see also [16, 18; no symmetry assumptions need to be imposed on $A$. The $S$-invariance of $H$ and the fact that the generator of $S=S_{H}$ admits a bounded $H^{\infty}$-calculus are trivial. Therefore, (3) is satisfied again. Note that the domain characterisation reduces to $\mathrm{D}_{p}(L)=\mathrm{D}_{p}\left(D^{2}\right)$, where $D$ is the derivative on $\mathbb{R}^{d}$. The techniques used in 27] to prove (1) are very different, involving diagonalisation arguments and the non-commuting Dore-Venni theorem. The identification of $\mathrm{D}_{p}(L)=\mathrm{D}_{p}\left(D^{2}\right)$ for $p=2$ had been obtained previously by Lunardi [23.

Our final corollary extends the characterisations of $\mathrm{D}_{p}(L)$ contained in Examples 2 and 3 and lifts the non-degeneracy assumption on $H$ in Example 3.

Corollary 3.6. If $S$ restricts to an analytic $C_{0}$-semigroup on $H$ which is contractive with respect to some equivalent Hilbert space norm, then for all $1<p<\infty$ we have

$$
\mathrm{D}_{p}(L)=\mathrm{D}_{p}\left(D_{H}^{2}\right) \cap \mathrm{D}_{p}\left(A_{\infty}^{*} D\right),
$$

where $D$ is the Malliavin derivative in the direction of $H_{\infty}$.

Proof. As has already been mentioned in the discussion preceding Theorem 3.4, the assumptions imply that $P$ is analytic. Moreover, since the restricted semigroup $S_{H}$ is similar to an analytic contraction semigroup, its negative generator $-A_{H}$ admits a bounded $H^{\infty}$-calculus, and the result follows from Theorem 3.5 .

Let us finally mention that the results in [25] have been proved for a more general class of elliptic operators on Wiener spaces (cf. Section 3 of that paper). In this setting the data consist of

- an arbitrary Gaussian measure $\mu$ on a separable Banach space $E$ with reproducing kernel Hilbert space $\mathscr{H}$

- an analytic $C_{0}$-contraction semigroup $\mathscr{S}$ on $\mathscr{H}$ with generator $\mathscr{A}$.

Given these data, the semigroup $\mathscr{P}$ is defined on $L^{2}(E, \mu)$ by second quantisation of the semigroup $\mathscr{S}$. Roughly speaking, this means that one uses the Wiener-Itô isometry to identify $L^{2}(E, \mu)$ with the symmetric Fock space over $\mathscr{H}$, i.e., the direct sum of symmetric tensor powers of $\mathscr{H}$. The semigroup $\mathscr{P}$ is then defined by applying $\mathscr{S}$ to each factor

$$
\mathscr{P}(t) \sum_{\sigma \in S_{n}}\left(h_{\sigma(1)} \otimes \ldots \otimes h_{\sigma(n)}\right):=\sum_{\sigma \in S_{n}} \mathscr{S}(t) h_{\sigma(1)} \otimes \ldots \otimes \mathscr{S}(t) h_{\sigma(n)},
$$

where $S_{n}$ is the permutation group on $\{1, \ldots, n\}$. For the details of this construction we refer to 19 . Equivalently, the semigroup $\mathscr{P}$ can be defined via the the following generalisation of the classical Mehler formula,

$$
\mathscr{P}(t) f(x)=\int_{E} f\left(\mathscr{S}(t) x+\sqrt{I-\mathscr{S}^{*}(t) \mathscr{S}(t)} y\right) d \mu(y),
$$

which makes sense by virtue of the fact that any bounded linear operator on $\mathscr{H}$ admits a unique measurable linear extension to $E[\underline{3}$. The generator $\mathscr{L}$ of the semigroup $\mathscr{P}$ is the elliptic operator formally given by

$$
\mathscr{L}=D^{*} \mathscr{A} D,
$$


where $D$ denotes the Malliavin derivative associated with $\mu$ and its adjoint $D^{*}$ is the associated divergence operator. The application to Ornstein-Uhlenbeck operators described in this paper is obtained by taking $\mu \sim \mu_{\infty}$ and $\mathscr{A} \sim A_{\infty}^{*}$ (cf. [5, 28]).

\section{An eXAmple}

In this section we present an example of a Hilbert space $E$, a continuously embedded Hilbert subspace $H \hookrightarrow E$, and a $C_{0}$-semigroup generator $A$ on $E$ such that:

- the semigroup $S$ generated by $A$ fails to be analytic;

- the stochastic Cauchy problem

$$
d U(t)=A U(t) d t+d W_{H}(t)
$$

admits a unique invariant measure, which we denote by $\mu_{\infty}$;

- the associated Ornstein-Uhlenbeck semigroup $P$ is analytic on $L^{2}\left(E, \mu_{\infty}\right)$.

Thus, although analyticity of $P$ implies analyticity of $S_{H}$ (Theorem 3.3), it does not imply analyticity of $S$.

Let $E=L^{2}\left(\mathbb{R}_{+}, e^{-x} d x\right)$ be the space of all measurable functions $f$ on $\mathbb{R}_{+}$such that

$$
\|f\|:=\left(\int_{0}^{\infty}|f(x)|^{2} e^{-x} d x\right)^{\frac{1}{2}}<\infty .
$$

The rescaled left translation semigroup $S$,

$$
S(t) f(x):=e^{-t} f(x+t), \quad f \in E, t>0, x>0
$$

is strongly continuous and contractive on $E$, and satisfies $\|S(t)\|=e^{-t / 2}$. Let $H=H^{2}\left(\mathbb{C}_{+}\right)$be the Hardy space of analytic functions $g$ on the open right-half plane $\mathbb{C}_{+}=\{z \in \mathbb{C}: \operatorname{Re} z>0\}$ such that

$$
\|g\|_{H}:=\sup _{x>0}\left(\int_{-\infty}^{\infty}|g(x+i y)|^{2} d y\right)^{\frac{1}{2}}<\infty .
$$

Since $\lim _{x \rightarrow+\infty} g(x)=0$ for all $g \in H$, the restriction mapping $i:\left.g \mapsto g\right|_{\mathbb{R}_{+}}$ is well-defined as a bounded operator from $H$ to $E$. By uniqueness of analytic continuation, this mapping is injective. Since $i$ factors through $L^{\infty}\left(\mathbb{R}_{+}, e^{-x} d x\right)$, $i$ is Hilbert-Schmidt [29, Corollary 5.21]. As a consequence (see, e.g., [9, Chapter $11]$ ), the Cauchy problem $d U(t)=A U(t) d t+d W_{H}(t)$ admits a unique invariant measure $\mu_{\infty}$.

The rescaled left translation semigroup $S_{H}$,

$$
S_{H}(t) g(z):=e^{-t} g(z+t), \quad f \in H, t \geqslant 0, \operatorname{Re} z>0
$$

is strongly continuous on $H$, it extends to an analytic contraction semigroup of angle $\frac{1}{2} \pi$, and satisfies $\left\|S_{H}(t)\right\|_{H}=e^{-t / 2}$. Clearly, for all $t \geqslant 0$ we have $S(t) \circ i=i \circ S_{H}(t)$. By these observations combined with [18, Theorem 9.2], the associated OrnsteinUhlenbeck semigroup $P$ is analytic. 


\section{Application to the stochastic heat equation}

In this final section we shall apply our results to the following stochastic PDE with additive space-time white noise:

$$
\begin{aligned}
\frac{\partial u}{\partial t}(t, y) & =\frac{\partial^{2} u}{\partial y^{2}}(t, y)+\frac{\partial^{2} W}{\partial t \partial y}(t, y), & & t \geqslant 0, y \in[0,1], \\
u(t, 0) & =u(t, 1)=0, & & t \geqslant 0, \\
u(0, y) & =0, & y & \in[0,1] .
\end{aligned}
$$

This equation can be cast into the abstract form (SCP) by taking $H=E=L^{2}(0,1)$ and $A$ the Dirichlet Laplacian $\Delta$ on $E$. The resulting equation

$$
\begin{aligned}
d U(t) & =A U(t) d t+d W(t), \\
U(0) & =0,
\end{aligned}
$$

where now $W$ denotes an $H$-cylindrical Brownian motion, has a unique solution $U$ given by

$$
U(t)=\int_{0}^{t} S(t-s) d W(s), \quad t \geqslant 0
$$

where $S$ denotes the heat semigroup on $E$ generated by $A$. Let $\mu_{\infty}$ denote the unique invariant measure on $E$ associated with $U$, and let $H_{\infty}$ denote its reproducing kernel Hilbert space. Let $i_{\infty}: H_{\infty} \hookrightarrow E$ denote the canonical embedding and let $i: H \rightarrow E$ be the identity mapping. By [17, Theorem 3.5, Corollary 5.6] the densely defined operator $V: i_{\infty}^{*} x^{*} \mapsto i^{*} x^{*}$ defined in (3.1) is closable from $H_{\infty}$ to $H$.

Let $L$ be the generator of the Ornstein-Uhlenbeck semigroup $P$ on $L^{p}\left(E, \mu_{\infty}\right)$ associated with $U$. Since $P$ is analytic, the results of Sections 2 and 3 can be applied. Noting that $\Delta$ is selfadjoint on $H$, condition (3) of Theorem 3.5 is satisfied and therefore

$$
\mathrm{D}_{p}(\sqrt{-L})=\mathrm{D}_{p}(D) \quad(1<p<\infty)
$$

where $D=D_{H}=D_{E}$ denotes the Fréchet derivative on $L^{p}\left(E, \mu_{\infty}\right)$.

One can go a step further by noting that the problem (5.1) is well-posed even on the space

$$
\widetilde{E}:=C_{0}[0,1]=\{f \in C[0,1]: f(0)=f(1)=0\},
$$

in the sense that the random variables $U(t)$ are $\widetilde{E}$-valued almost surely and that $U$ admits has a modification $\widetilde{U}$ with continuous (in fact, even Hölder continuous) trajectories in $\widetilde{E}$. Moreover, the invariant measure $\mu_{\infty}$ is supported on $\widetilde{E}$. In analogy to (1.1) this allows us to define an "Ornstein-Uhlenbeck semigroup" $\widetilde{P}$ on $L^{p}\left(\widetilde{E}, \mu_{\infty}\right)$ associated with $\widetilde{U}$ by

$$
\widetilde{P}(t) f(x):=\mathbb{E} f\left(\widetilde{U}^{x}(t)\right), \quad t \geqslant 0, x \in \widetilde{E},
$$

where $\widetilde{U}^{x}(t)=\widetilde{S}(t) x+\widetilde{U}(t)$ and $\widetilde{S}$ is the heat semigroup on $\widetilde{E}$. It is important to observe that we are not in the framework considered in the previous sections, due to the fact that $H=L^{2}(0,1)$ is not continuously embedded in $\tilde{E}$. Let $\widetilde{L}$ denote the generator of $\widetilde{P}$. Under the natural identification

$$
L^{p}\left(\widetilde{E}, \mu_{\infty}\right)=L^{p}\left(E, \mu_{\infty}\right)
$$


(using that the underlying measure spaces are identical up to a set of measure zero), we have $\widetilde{P}(t)=P(t)$ and $\widetilde{L}=L$, so that

$$
\mathrm{D}_{p}(\sqrt{-\widetilde{L}})=\mathrm{D}_{p}(\sqrt{-L})=\mathrm{D}_{p}(D) \quad(1<p<\infty) .
$$

This representation may seem somewhat unsatisfactory, as the right-hand side refers explicitly to the ambient space $E$ in which $\widetilde{E}$ is embedded. An intrinsic representation of $\mathrm{D}_{p}(\sqrt{-\widetilde{L}})$ can be obtained as follows. For functions $F: \widetilde{E} \rightarrow \mathbb{R}$ of the form

$$
F(f)=\phi\left(\int_{0}^{1} f g_{1} d t, \ldots, \int_{0}^{1} f g_{N} d t\right), \quad f \in \widetilde{E},
$$

with $\phi \in C_{\mathrm{b}}^{2}\left(\mathbb{R}^{N}\right)$ and $g_{1}, \ldots, g_{N} \in H$, we define $\widetilde{D} F: \widetilde{E} \rightarrow H$ by

$$
\widetilde{D} F(f)=\sum_{n=1}^{N} \frac{\partial \phi}{\partial y_{n}}\left(\int_{0}^{1} f g_{1} d t, \ldots, \int_{0}^{1} f g_{N} d t\right) g_{n}, \quad f \in \widetilde{E} .
$$

This operator is closable in $L^{p}\left(\widetilde{E}, \mu_{\infty}\right)$ for all $1 \leqslant p<\infty$. On $L^{2}\left(\widetilde{E}, \mu_{\infty}\right)$ we have the representation

$$
\widetilde{L}=\widetilde{D}^{*} \widetilde{D}
$$

As a result we can apply [25, Theorem 2.1] directly to the operator $V$ and obtain that

$$
\mathrm{D}_{p}(\sqrt{-\widetilde{L}})=\mathrm{D}_{p}(\widetilde{D}) \quad(1<p<\infty) .
$$

This answers a question raised by Zdzisław Brzeźniak (personal communication). To make the link between the formulas (5.2) and (5.3) note that, under the identification $L^{p}\left(\widetilde{E}, \mu_{\infty}\right)=L^{p}\left(E, \mu_{\infty}\right)$, one also has $\mathrm{D}_{p}(\widetilde{D})=\mathrm{D}_{p}(D)$.

Remark 5.1. It is possible to give explicit representations for the space $H_{\infty}$ and the operator $V$. To begin with, the covariance operator $Q_{\infty}$ of $\mu_{\infty}$ is given by

$$
Q_{\infty} f=\int_{0}^{\infty} S(t) S^{*}(t) f d t=\int_{0}^{\infty} S(2 t) f d t=\frac{1}{2} \Delta^{-1} f, \quad f \in E .
$$

It follows that the reproducing kernel Hilbert space $H_{\infty}$ associated with $\mu_{\infty}$ equals

$$
H_{\infty}=\mathrm{R}\left(\sqrt{Q_{\infty}}\right)=\mathrm{D}(\sqrt{-\Delta})=H_{0}^{1}(0,1) .
$$

Noting that $Q_{\infty}=i_{\infty} \circ i_{\infty}^{*}$, we see that the operator $V: i_{\infty}^{*} x^{*} \mapsto i^{*} x^{*}$ is given by

$$
\begin{gathered}
\mathrm{D}(V)=H^{2}(0,1) \cap H_{0}^{1}(0,1), \\
V f=2 \Delta f, \quad f \in \mathrm{D}(V) .
\end{gathered}
$$

Remark 5.2. Formulas for $\mathrm{D}_{p}(\widetilde{L})$ analogous to (5.2) and (5.3) can be deduced from Theorem 3.5 and [25, Theorem 2.2] in a similar way.

The Ornstein-Uhlenbeck operators $L$ and $\widetilde{L}$ considered above are symmetric on $L^{2}\left(E, \mu_{\infty}\right)$, and therefore the domain identifications for their square roots could essentially be obtained from the results of [6, 32. The above argument, however, can be applied to a large class of second order elliptic differential operators $A$ on $L^{2}(0,1)$ (but explicit representations as in Remark 5.1 are only possible when $A$ is selfadjoint).

In fact, under mild assumptions on the coefficients and under various types of boundary conditions, such operators $A$ have a bounded $H^{\infty}$-calculus on $H=E=$ 
$L^{2}(0,1)$ (see [11, 14, 20] and there references therein). By the result of Le Merdy 22. mentioned earlier, this implies that the analytic semigroup $S$ generated by $A$ is contractive in some equivalent Hilbertian norm. Hence, by [18, Theorem 9.2], the associated Ornstein-Uhlenbeck semigroup is analytic. Typically, under Dirichlet boundary conditions, $S$ is uniformly exponentially stable. This implies (see 9]) that the solution $U$ of (SCP) admits a unique invariant measure. Finally, the analyticity of $S$ typically implies space-time Hölder regularity of $U$ (see [4, 13]), so

that the corresponding stochastic PDE is well-posed in $\widetilde{E}=C_{0}[0,1]$. We plan to provide more details in a forthcoming publication.

\section{REFERENCES}

[1] P. Auscher, A. M $\mathrm{M}^{\mathrm{C}} \mathrm{InTosh}$, And A. NAhmod, Holomorphic functional calculi of operators, quadratic estimates and interpolation, Indiana Univ. Math. J. 46 (1997), no. 2, 375-403.

[2] A. Axelsson, S. Keith, AND A. M ${ }^{\mathrm{C}} \mathrm{Intosh}$, Quadratic estimates and functional calculi of perturbed Dirac operators, Invent. Math. 163 (2006), no. 3, 455-497.

[3] V.I. Bogachev, "Gaussian Measures", Mathematical Surveys and Monographs, vol. 62, American Mathematical Society, Providence, RI, 1998.

[4] Z. BrZeŹNIAK, On stochastic convolution in Banach spaces and applications, Stochastics Stochastics Rep. 61 (1997), no. 3-4, 245-295.

[5] A. Chojnowska-Michalik and B. Goldys, Nonsymmetric Ornstein-Uhlenbeck semigroup as second quantized operator, J. Math. Kyoto Univ. 36 (1996), no. 3, 481-498.

[6] A. Chojnowska-Michalik and B. Goldys, Generalized Ornstein-Uhlenbeck semigroups: Littlewood-Paley-Stein inequalities and the P.A. Meyer equivalence of norms, J. Funct. Anal. 182 (2001), no. 2, 243-279.

[7] A. Chojnowska-Michalik And B. Goldys, Symmetric Ornstein-Uhlenbeck semigroups and their generators, Probab. Theory Related Fields 124 (2002), no. 4, 459-486.

[8] G. DA Prato, Characterization of the domain of an elliptic operator of infinitely many variables in $L^{2}(\mu)$ spaces, Atti Accad. Naz. Lincei Cl. Sci. Fis. Mat. Natur. Rend. Lincei (9) Mat. Appl. 8 (1997), no. 2, 101-105.

[9] G. Da Prato and J. ZabCZYK, "Stochastic Equations in Infinite Dimensions", Encyclopedia of Mathematics and its Applications, vol. 44, Cambridge University Press, Cambridge, 1992.

[10] G. Da Prato and J. Zabczyk, "Second Order Partial Differential Equations in Hilbert Spaces", London Mathematical Society Lecture Note Series, vol. 293, Cambridge University Press, Cambridge, 2002.

[11] R. Denk, G. Dore, M. Hieber, J. Prüss, and A. Venni, New thoughts on old results of R. T. Seeley, Math. Ann. 328 (2004), no. 4, 545-583.

[12] R. Denk, M. Hieber, And J. Prüss, R-boundedness, Fourier multipliers and problems of elliptic and parabolic type, Mem. Amer. Math. Soc. 166 (2003), no. 788.

[13] J. Dettweiler, J.M.A.M. van NeERven, And L. Weis, Space-time regularity of solutions of the parabolic stochastic Cauchy problem, Stoch. Anal. Appl. 24 (2006), no. 4, 843-869.

[14] X.T. Duong And A. McIntosh, Functional calculi of second-order elliptic partial differential operators with bounded measurable coefficients, J. Geom. Anal. 6 (1996), no. 2, 181-205.

[15] M. Fuhrman, Analyticity of transition semigroups and closability of bilinear forms in Hilbert spaces, Studia Math. 115 (1995), no. 1, 53-71.

[16] B. Goldys, On analyticity of Ornstein-Uhlenbeck semigroups, Atti Accad. Naz. Lincei Cl. Sci. Fis. Mat. Natur. Rend. Lincei (9) Mat. Appl. 10 (1999), no. 3, 131-140.

[17] B. Goldys, F. Gozzi, And J.M.A.M. van NeErven, On closability of directional gradients, Potential Anal. 18 (2003), no. 4, 289-310.

[18] B. Goldys And J.M.A.M. VAn NeERven, Transition semigroups of Banach space-valued Ornstein-Uhlenbeck processes, Acta Appl. Math. 76 (2003), no. 3, 283-330, updated version on arXiv:math/0606785.

[19] S. Janson, "Gaussian Hilbert Spaces", Cambridge Tracts in Mathematics, vol. 129, Cambridge University Press, Cambridge, 1997.

[20] N.J. Kalton, P.C. Kunstmann, And L. Weis, Perturbation and interpolation theorems for the $H^{\infty}$-calculus with applications to differential operators, Math. Ann. 336 (2006), no. 4, $747-801$. 
[21] P.C. Kunstmann and L. Weis, Maximal $L_{p}$-regularity for parabolic equations, Fourier multiplier theorems and $H^{\infty}$-functional calculus, in: "Functional Analytic Methods for Evolution Equations", Lecture Notes in Math., vol. 1855, Springer, Berlin, 2004, pp. 65-311.

[22] C. Le Merdy, The similarity problem for bounded analytic semigroups on Hilbert space, Semigroup Forum 56 (1998), no. 2, 205-224.

[23] A. Lunardi, On the Ornstein-Uhlenbeck operator in $L^{2}$ spaces with respect to invariant measures, Trans. Amer. Math. Soc. 349 (1997), no. 1, 155-169.

[24] J. MaAs And J.M.A.M. VAn NeERVEn, On analytic Ornstein-Uhlenbeck semigroups in infinite dimensions, Archiv Math. (Basel) 89 (2007), 226-236.

[25] J. MaAs And J.M.A.M. van NeERven, Boundedness of Riesz transforms for elliptic operators on abstract Wiener spaces, J. Funct. Anal. 257 (2009), no. 8, 2410-2475.

[26] A. Mc Intosh And A. YAGI, Operators of type $\omega$ without a bounded $H_{\infty}$ functional calculus, "Miniconference on Operators in Analysis" (Sydney, 1989), Proc. Centre Math. Anal. Austral. Nat. Univ., vol. 24, Austral. Nat. Univ., Canberra, 1990, pp. 159-172.

[27] G. Metafune, J. Prüss, A. Rhandi, and R. Schnaubelt, The domain of the OrnsteinUhlenbeck operator on an $L^{p}$-space with invariant measure, Ann. Sc. Norm. Super. Pisa Cl. Sci. (5) 1 (2002), no. 2, 471-485.

[28] J.M.A.M. VAN NeERVEn, Nonsymmetric Ornstein-Uhlenbeck semigroups in Banach spaces, J. Funct. Anal. 155 (1998), no. 2, 495-535.

[29] J.M.A.M. van NeERven, "Stochastic Evolution Equations", 2008, Lecture Notes of the 11th Internet Seminar, TU Delft OpenCourseWare, http://ocw.tudelft.nl.

[30] J.M.A.M. Van NeERven AND L. Weis, Stochastic integration of functions with values in a Banach space, Studia Math. 166 (2005), no. 2, 131-170.

[31] D. Nualart, "The Malliavin Calculus and Related Topics", second ed., Probability and its Applications, Springer-Verlag, Berlin, 2006.

[32] I. ShigeKaWA, Sobolev spaces over the Wiener space based on an Ornstein-Uhlenbeck operator, J. Math. Kyoto Univ. 32 (1992), no. 4, 731-748.

Institute for Applied Mathematics, University of Bonn, Endenicher Allee 60, 53115 Bonn, Germany

E-mail address: maas@iam.uni-bonn.de

Delft Institute of Applied Mathematics, Delft University of Technology, P.O. Box 5031, 2600 GA Delft, The Netherlands

E-mail address: J.M.A.M.vanNeerven@TUDelft.nl 